\title{
Accuracy of the approximate expressions for pure Cotton-Mouton effect from JET Real-Time Far Infrared Polarimeter
}

\author{
Bohdan Bieg ${ }^{1}$ \\ ${ }^{I}$ Maritime University of Szczecin, Waly Chrobrego 1-2, 70-500 Szczecin, Poland \\ E-mail: b.bieg@am.szczecin.pI
}

\author{
Alexandru Boboc ${ }^{2}$ \\ ${ }^{2}$ CCFE, Culham Science Centre, Abingdon, OX14 3DB, UK \\ and JET Contributors ${ }^{3,4}$ \\ ${ }^{3}$ EUROfusion Consortium, JET, Culham Science Centre, Abingdon, OX14 3DB, UK \\ ${ }^{4}$ See the Appendix of F. Romanelli et al., Proceedings of the 25th IAEA Fusion Energy Conference 2014, \\ Saint Petersburg, Russia
}

The JET Far-Infrared (FIR) polarimeter is a laser based instrument used for determining the poloidal magnetic field via the Faraday rotation angle measurements and more recently, for determining the line-integrated electron plasma density (LID) via the Cotton-Mouton ellipticity angle measurements. However, at high plasma current and plasma density the Cotton-Mouton effect combines nonlinearly with the Faraday effect, hampering an accurate determination. There have been several investigations in the last decade dedicated to the subject, but all were carried out for different plasma regimes and conditions. We take a different approach, where different approximations for estimating the line-integrated plasma density by polarimetry are analysed in a wide variety of conditions, representative of the JET operating domain, focusing in particular on pulses with high plasma current, high density and high magnetic field. This new set of data will be compared with the line-integrated measurements obtained by other means, such as interferometry and high resolution Thomson scattering. A complete sensitivity study of the various formulations for LID from polarimetry will be performed to analyse the potential of these formulations for complementing the interferometry measurements with the ultimate goal of providing fringe-jump free LID measurements on JET during future experimental campaigns.

First EPs Conference on Plasma Diagnostics - $1^{\text {st }}$ ECPD

14-17 April 2015,

Villa Mondragone, Frascati (Rome) Italy

\footnotetext{
${ }^{1}$ Speaker 


\section{Introduction}

The electron density is one of the main plasma parameters, crucial of any fusion devices such as the Tokamak and the Stellarator. The standard diagnostic for the electron line-integrated density (LID) measurement at JET is Far-Infrared (FIR) interferometer, method integrated in the real-time control systems and proved for many years. Despite the reliability of interferometry, there are cases when LID measurement is corrupted or even lost due various effects such as mechanical vibrations, refraction of FIR beams, or when the rate of change of the plasma density is faster than the interferometry sampling rate due to turbulent effects such as Edge Localised Modes, disruptions, impurity seeding or when pellets are injected into the plasma for example. The installation of JET's ITER-like metallic wall has caused new types of diagnostic issues, that heavily affect many diagnostic systems including interferometry. The loss of the FIR signal generates the so called fringe jumps in the measured LID, each leading to an uncertainty in the measured line-integrated density value of $1.143 \times 10^{-19} \mathrm{~m}^{-2}$. In the case of JET, a backup measurement was proposed, based on LID evaluation using polarimetric the Cotton-Mouton effect (CM) [1]. This effect consists in the change of the electromagnetic beam ellipticity and takes place when the beam passes through birefringent medium with transverse magnetic field, like magnetized plasma. In the first approximation, assuming that the toroidal filed $B_{t}$ is the only one component of the magnetic field vector, the total change in the ellipticity angle $\chi$, characterizing the beam ellipticity (defined in literature as $\varepsilon=\tan (\chi)$ ), is proportional to the line-integrated density $n_{e}$ of the plasma as follows:

$$
\Delta \chi=\frac{1}{2} C_{1} \lambda^{3} \cdot \int n_{e} B_{t}^{2} \mathrm{~d} z
$$

where $C_{1}=2.4565 \cdot 10^{-11} \mathrm{rad} / \mathrm{mT}^{2}$ and $\lambda$ is the electromagnetic beam wavelength.

For the JET vertical lines of sight $B_{t}$ is nearly constant along the path and the line-integrated and the line-integrated plasma density could be very simply determined from the change in the value of the ellipticity angle alone:

$$
\operatorname{LID}_{\chi}=\int n_{e} \mathrm{~d} z=\frac{2 \Delta \chi}{C_{1} \lambda^{3} B_{t}^{2}} .
$$

Unfortunately the presence of the poloidal magnetic field, with components of the magnetic field perpendicular and parallel to the beam, leads to a more complex evolution of the beam polarization state. The small perpendicular component $B_{r}$ slightly modify the change in the ellipticity angle caused by strong toroidal field $B_{t}$. The magnetic field component $B_{z}$, parallel to the beam propagation direction, brings on the polarization plane rotation (the Faraday effect), measured as a change in the value of the polarization angle $\psi$. Additionally, there is nonlinear mutual coupling of both polarization angles - the rate of polarization angle rotation is affected by the value of the ellipticity angle $\chi$, and vice versa. The evolution of the polarization state $(\psi, \chi)$ of the laser beam propagating through the magnetized plasma without dissipation, in cold plasma approximation can be described by the set of two differential equations [2]: 


$$
\left\{\begin{array}{l}
\frac{\mathrm{d} \psi}{\mathrm{d} z}=\frac{1}{2} \Omega_{3}-\frac{1}{2}\left(\Omega_{1} \cos 2 \psi+\Omega_{2} \sin 2 \psi\right) \tan 2 \chi \\
\frac{\mathrm{d} \chi}{\mathrm{d} z}=\frac{1}{2}\left(\Omega_{1} \sin 2 \psi-\Omega_{2} \cos 2 \psi\right)
\end{array}\right.
$$

where the terms $\Omega_{1}=2.4565 \cdot 10^{-11} \lambda^{3} n_{e} \cdot\left(B_{t}^{2}-B_{r}^{2}\right)$ and $\Omega_{2}=2.4565 \cdot 10^{-11} \lambda^{3} n_{e} \cdot 2 B_{t} B_{r}$ account for the Cotton-Mouton effect, while $\Omega_{3}=5.2624 \cdot 10^{-13} \lambda^{2} n_{e} \cdot B_{z}$ accounts for the Faraday rotation effect [5]. From the equations (3) it is clear that equations (1) and (2) are accurate only in the case when along the whole path the polarization angle is equal to the initial value $\psi_{0}=\pi / 4$ and when constant $B_{t}$ is the only one magnetic field component - so $\Omega_{2}=\Omega_{3}=0$. In current JET plasma conditions none of these conditions is fulfilled: the Faraday rotation angle $\Delta \psi$ sometimes exceeds 1.2 radians (or 70 degrees), toroidal magnetic field component $B_{t}$ varies along the path and $\left|\Omega_{3}\right|>\Omega_{1}>>\Omega_{2}$.

In order to estimate the LID value from the polarimetry several approximations have been proposed, consist in obtaining from polarimetry data $(\psi, \chi)$ such a quantity $W_{1}$ that is defined as follows:

$$
\mathrm{LID}=\int n_{e} \mathrm{~d} z=\frac{W_{1}}{C_{1} \lambda^{3} B_{t}^{2}}
$$

Here we present the statistical analysis of five different models proposed in the literature, using measurements from various plasma scenarios representative of the JET operating domain focusing in particular on pulses with high plasma current, high density and high magnetic field. All analysed polarimetry data were obtained using new calibration method, introduced to JET polarimetry system in 2014 [see ref.3]. This set of data was compared with the line-integrated measurements obtained by interferometry that is considered the reference for LID. The final aim was to evaluate the potential of each formulation for complementing the interferometry measurements with the ultimate goal of providing fringe-jump free LID measurements used for density fuelling control and machine protection on JET during future experimental campaigns.

\section{Evaluation of the line-integrated density}

Line-integrated electron plasma density from polarimetry could be evaluated by five methods, described in details in references [4-6]. Three simplest methods assume that both the the Faraday and the Cotton-Mouton effects are small and the mutual coupling between $\chi$ and $\psi$ are only of the second-order effect. In these conditions the equation (2) is a good approximation of the rigorous solution of equations (3) and therefore for the initial polarization state $\left(\psi_{0}, \chi_{0}\right)=(\pi / 4,0)$ parameter $W_{1}$ is equal to doubled final ellipticity angle:

$$
W_{1 \chi}=2 \chi
$$

Next two models comes from fact that the ellipticity of the beam could also be described by the phase shift angle $\delta$ or Stokes vector component $s_{3}$. These two quantities are related to the polarization angles $\psi$ and $\chi$ by formulas $\tan \delta=\tan 2 \chi / \sin 2 \psi$ and $s_{3}=\sin 2 \chi$, which in the case of the initial polarization state $\left(\psi_{0}, \chi_{0}\right)=(\pi / 4,0)$ and weak Faraday and Cotton-Mouton 
effect, simplify to $\delta=2 \chi$ and $s_{3}=2 \chi$. Thus next two approximations for $W_{1}$ parameter take a form

$$
\begin{aligned}
& W_{1 \delta}=\delta \\
& W_{1 s}=s_{3}
\end{aligned}
$$

Two more sophisticated expressions for parameter $W_{1}$ has been proposed in [4] and written in slightly different form in [5]. These two models come from two opposite assumptions:

A) $\Omega_{3} / \Omega_{1}$ ratio is constant along the whole path;

B) $\Omega_{3} / \Omega_{1}$ ratio is equal zero at the beginning and at the end of laser path into the plasma (pure Cotton-Mouton effect takes place), and tends to infinity at central part of laser path (pure Faraday rotation takes place)

In both cases $\Omega_{2}=0$ is assumed. A detailed description of these two models can be found in the references $[4,5]$ and here only refer to the simplified final form, adjusted to JET polarimetry conditions $\left.\left(\left(\psi_{0}, \chi_{0}\right)=(\pi / 4,0), B_{t}>B_{r}, \Delta \psi, \Delta \chi<\pi / 4\right)\right)$ :

$$
\begin{aligned}
& W_{1 A}=\arcsin \left(\sqrt{s_{1}^{2}+s_{3}^{2}}\right) / \sqrt{1+\left(\frac{s_{1}}{s_{3}}\right)^{2}} \\
& W_{1 B}=2 \arctan \left(\frac{s_{3}}{1+s_{2}}\right)
\end{aligned}
$$

where $s_{i}$ are Stokes vector components: $s_{1}=\cos (2 \chi) \cos (2 \psi), \quad s_{2}=\cos (2 \chi) \sin (2 \psi)$ and $s_{3}=\sin 2 \chi$. However it should be noted that neither the conditions set at model A or model B are not strictly complied with in the case of JET.

In our statistical analysis we evaluated the LID from the five models described above as $\mathrm{LID}_{\chi}, \mathrm{LID}_{\delta}, \mathrm{LID}_{s}, \mathrm{LID}_{A}, \mathrm{LID}_{B}$, were interferometry LID is defined, for consistency, as $\mathrm{LID}_{\text {int }}$

\section{Statistical analysis}

The JET FIR diagnostic has 8 channels, 4 vertical and 4 laterals [5]. In the present work we focus on the data provided by the FIR diagnostic core channel (vertical channel 3) only as is the one used for machine protection and density feedback control. The statistical analysis was performed on high performance, 40 JET pulses obtained in July-August 2014, with maximum values: LID measured by interferometer $-2.7 \cdot 10^{20} \mathrm{~m}^{-2}$, plasma current $-4.0 \mathrm{MA}$, toroidal magnetic field $-3.7 \mathrm{~T}$, the Faraday rotation variation $-0.55 \mathrm{rad}$ and the Cotton Mouton effect $0.24 \mathrm{rad}$. The methodology for the analysis was as follows: for each shot we selected several hundred of temporal points and evenly distributed over pulse length, all adding to a total of 15000 measurement points for the selected database.

Figure 1 shows an example of the comparison of the line-integrated density calculated from polarimetry data and equations (4) - (9) to the interferometric line-integrated density interferometry for pulse 87386. At low plasma densities all approximations give the same LID value. At high plasma densities the best agreement with LID measured by interferometry is for the model assuming constant relative strength of the Faraday and the Cotton-Mouton effects. For almost all analysed temporal points within 87386 shot the difference 
$\Delta \mathrm{LID}_{A}=\mathrm{LID}_{A}-\mathrm{LID}_{\text {int }}$ is below one fringe. In this particular example, best agreement between LID from interferometry and polarimetry could be obtain within model "A".
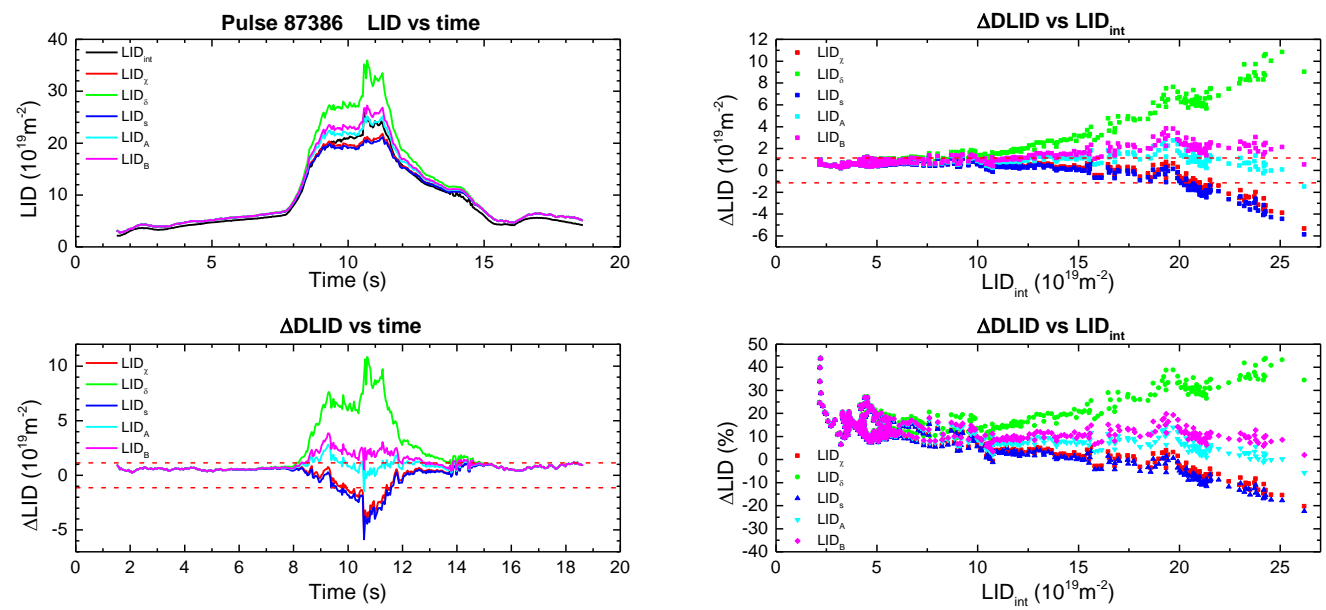

Figure 1. Comparison of line-integrated density $L D_{\text {int }}$ obtained from interferometry with line integrated densities calculated from five examined approximations (dashed red lines are the threshold of one fringe $1.143 \cdot 10^{19} \mathrm{~m}^{-2}$ )

The statistical comparison between various polarimetry models for LID and interferometry are depicted in the Figure 2. In all analysed models at high plasma densities there are cases when the maximum discrepancy with respect interferometry is over 3 fringes (about 5-10\%). To minimize the difference between line-integrated density value from polarimetry and interferometry, additional model could be proposed for JET channel 3 based on the analysis of empirical data and introducing additional calibration factor such as described below:

$$
W_{1 B 1}=W_{1 B} / 1.1
$$

Within this simple mathematical correction, at high plasma densities, the agreement between polarimetry and interferometry in evaluating LID reduces to 1.5 fringe range. A histogram of the results obtained by the simplest model $\mathrm{LID}_{\chi}$ and empirical model $\mathrm{LID}_{\mathrm{B} 1}$ with respect to $\mathrm{LID}_{\text {int }}$ is depicted on Figure 3 and associated Table 1 .
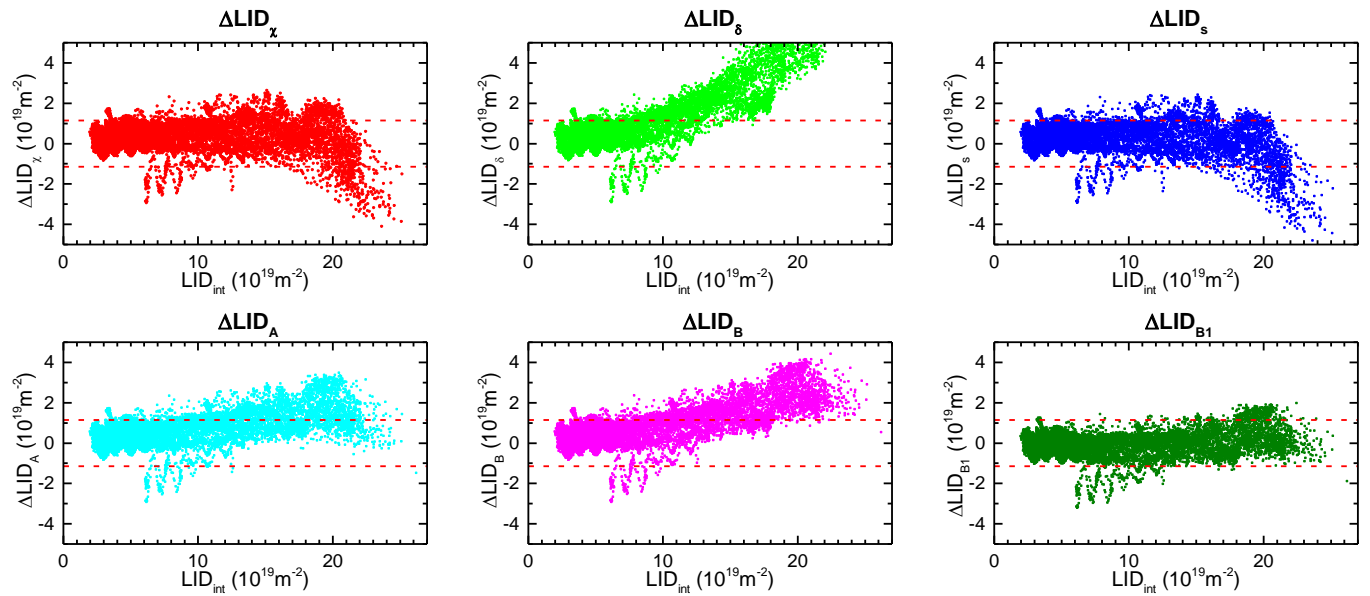

Figure 2. Statistical analysis of the polarimetry LIDs obtained by the five approximation models and the LID density as measured by the interferometer 

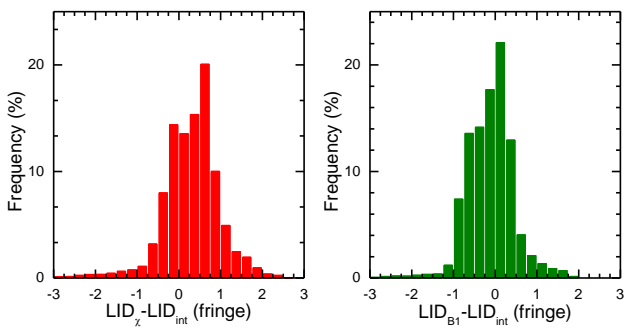

Figure 3. Histogram of difference between $\mathrm{LID}_{\chi}$ and $\mathrm{LID}_{\mathrm{B} 1}$ with respect to $\mathrm{LID}_{\text {int }}$

\begin{tabular}{|c|c|c|}
\hline $\begin{array}{c}\text { LID-LID } \\
\text { (fring })\end{array}$ & $\begin{array}{c}\mathrm{LID}_{\chi} \\
(\%)\end{array}$ & $\begin{array}{c}\mathrm{LID}_{B 1} \\
(\%)\end{array}$ \\
\hline$[-2,-1]$ & 2,2 & 2,2 \\
\hline$[-1,0]$ & 26,7 & 53,3 \\
\hline$[0,1]$ & 59,0 & 41,2 \\
\hline$[1,2]$ & 10,3 & 3,1 \\
\hline
\end{tabular}

Table 1: Frequency count of difference between $\mathrm{LID}_{\chi}$ and $\mathrm{LID}_{\mathrm{B} 1}$ with respect to $\mathrm{LID}_{\mathrm{int}}$

\section{Conclusions}

Approximation $\mathrm{LID}_{\chi}$, currently used for real-time application at JET, as well as model $\mathrm{LID}_{\mathrm{B} 1}$ are in very good agreement with $\mathrm{LID}_{\text {int }}$ value in two fringe range $(98.2 \%$ and $99.8 \%$ respectively). However, in more rigorous range of one fringe error level, the advantage of empirical model $\mathrm{LID}_{\mathrm{B} 1}$ described by (10) becomes apparent as it is $95 \%$ the agreement with $\mathrm{LID}_{\text {int }}$, while approximation $\mathrm{LID}_{\chi}$, given by (2), gives only $86 \%$ agreement. At high plasma density range $\operatorname{LID}_{\chi}$ overestimate LID value up to 2.5 fringes for some pulses while for other underestimate up to 4 fringes.

Since both the ellipticity and the Faraday rotation angle are available in real time, the approximation $\mathrm{LID}_{\mathrm{B} 1}$, with an appropriate calibration factor, could be used as a better estimator for the line integrated electron density on JET machine in the view of use for fuelling control and machine protection. However this requires further analysis and development.

\section{Acknowledgement}

This work has been carried out within the framework of the EUROfusion Consortium and has received funding from the Euroatom research and training programme 2014-2018 under grant agreement No 633053. The views and opinions expressed herein do not necessarily reflect those of the European Commission

\section{References}

[1] Ch. Fuchs and H. J. Hartfuss, Line integrated density measurements based on Cotton-Mouton polarimetry, Rev. Sci. Instrum. 70 (1999) 722

[2] B.Bieg, J.Chrzanowski, Yu.A. Kravtsov, A. Murari, F. Orsitto, Two approaches to plasma polarimetry: angular variables technique and Stokes vector formalism, Nuclear Instruments \& Methods In Physics Research Section A 720 (2013) 157-159

[3] A. Boboc at all, A novel calibration method for JET Real-Time Far Infrared Polarimeter and integration of polarimetry based line-integrated density measurements for machine protection of a fusion plant, submitted to Rev. Sci. Instrum.

[4] K. Guenther and JET-EFDA Contributors, Approximate method to extract the pure Faraday and Cotton-Mouton effects from polarimetry measurements in a tokamak, Plasma Phys. Control. Fusion 46 (2004) 1423

[5] S. E. Segre, V. Zanza, Derivation of the pure Faraday and Cotton-Mouton effects when polarimetric effects in a tokamak are large, Plasma Phys. Controlled Fusion 48 (2006) 339

[6] F. P. Orsitto at all, modelling of polarimetry measurements at JET, Plasma Phys. Control. Fusion 50 (2008) 115009 